\title{
The Human Arm as a Redundant Manipulator: The Control of Path and Joint Angles
}

\author{
H. Cruse and M. Brüwer \\ Fakultät für Biologie, Universität Bielefeld, Universitätsstrasse, D-4800 Bielefeld 1, \\ Federal Republic of Germany
}

\begin{abstract}
The movements studied involved moving the tip of a pointer attached to the hand from a given starting point to a given end point in a horizontal plane. Three joints - the shoulder, elbow and wrist were free to move. Thus the system represented a redundant manipulator. The coordination of the movements of the three joints was recorded and analyzed. The study concerned how the joints are controlled during a movement. The results are used to evaluate several current hypotheses for motor control.

Basically, the incremental changes are calculated so as to move the tip of the manipulator along a straight line in the workspace. The values of the individual joints seem to be determined as follows. Starting from the initial values the incremental changes in the three joint angles represent a compromise between two criteria: 1) the amount of the angular change should be about the same in the three joints, and 2) the angular changes should minimize the total cost of the arm position as determined by cost functions defined for each joint as a function of angle.

By itself, this mechanism would produce strongly curved trajectories in joint space which could include additional acceleration and deceleration in a joint. These are reduced by the influence of a third criterion which fits with the mass-spring hypothesis. Thus the path is calculated as a compromise between a straight line in workspace and a straight line in joint space. The latter can produce curved paths in the workspace such as were actually found in the experiments.

A model calculation shows that these hypotheses can qualitatively describe the experimental findings.
\end{abstract}

\section{Introduction}

How the movernent of a manipulator with several joints is controlled is an interesting question for both biological and technological reasons. Such a manipulator may be redundant or non-redundant. A nonredundant manipulator has exactly as many joints as are necessary to solve a given task. A redundant manipulator has more joints than necessary. Thus a given point in the workspace of the manipulator can be reached by a number of different combinations of joint angles of the manipulator. The question arises of how the control system selects one of this infinite number of possible positions when trying to reach a given point. In an earlier paper considering the control of the human arm the following hypothesis was proposed (Cruse 1986). To each joint a cost function is attached which defines a cost value for each joint angle. The cost functions show a minimum at about the middle of the angle range of the joint and the cost values increase to either of the extreme angles. The total cost of a manipulator position is described as the sum of the actual cost values of all joints. When reaching to a given point in the workspace according to this hypothesis that manipulator position is selected out of the geometrically possible positions which shows the minimum total cost value. In this way the number of degrees of freedom of the system is reduced and thus the redundancy problem can be solved. However, the above investigation was restricted to a consideration of the static situation, i.e. only the position of the arm at the end point of the movement was examined. Consideration of the movement itself was neglected. In this paper the kinematics of the movement of the human arm will be considered. In the experiments described in Sects. 3.2 and 3.3 the start point and the end point of the tip of the manipulator in the workspace are given but the path of the tip of the manipulator, also called the position of the end effector, is left open to the subject. The time sequence of movement along the path will be presented only indirectly.

The questions investigated are, what kinds of paths are adopted? and is it possible to say something about 
the mechanisms which produce these paths? In particular, is the cost function principle able to explain the findings? In earlier investigations of planar arm movements only straight paths were reported (Morasso 1981; Soechting and Lacquaniti 1981; Abend et al. 1982). In contrast Atkeson and Hollerbach (1985) reported curved paths when investigating arm movements in the vertical plane. We will show in this paper that curved paths can also appear in planar movements and propose a model which is able to describe the observed movements.

\section{Methods}

As in the earlier paper (Cruse 1986) the movement of the arm is restricted to the horizontal plane and the three joints at shoulder, elbow and wrist are free to move. To measure the values of the joint angles the arm of the subject was laid on an artificial arm having three joints which was free to glide over a horizontal plane. The subjects arm could be clamped to the artificial arm so that the axes of rotation of the corresponding joints of both, the human and the artificial arm lay on the same axes. The part corresponding to the hand is prolonged by a pointer with a length of $28 \mathrm{~cm}$ and thus has about the same length as the upper and the lower arm. The joints of the artificial arm carried potentiometers to measure the values of the joint angles. These values could be read on a multichannel pen recorder (Gould ES 1000) or via $A-D$ converters directly into the computer (Apple II). In the first case the curves were later transferred to the computer by means of a graphic tablet. These values can be used to calculate and plot the graphs in the joint space. This is shown in two diagrams, each using the angle of the elbow joint as abscissa and the wrist and shoulder joint as ordinate, respectively. Furthermore the movement of the tip of the manipulator was calculated and shown in the (cartesian) workspace coordinates $x$ and $y$. The definition of the angles and the workspace coordinates can be found in Fig. 1. Because of lack of space the figures are limited to a few examples selected from all the movements investigated. Each figure consists of two columns. The left column shows the experimental results. The right column shows a direct comparison of the corresponding results from the model calculation. The details of the model will be discussed in the final section of this paper. It should be stressed that the definition of the elbow and the wrist angle used here differs from that used in the earlier paper (Cruse 1986).

The subjects were asked to make the movements in a "comfortable" way. Particularly, they were asked to move slowly rather than fast. This was done to eliminate inertia effects as much as possible. Move-

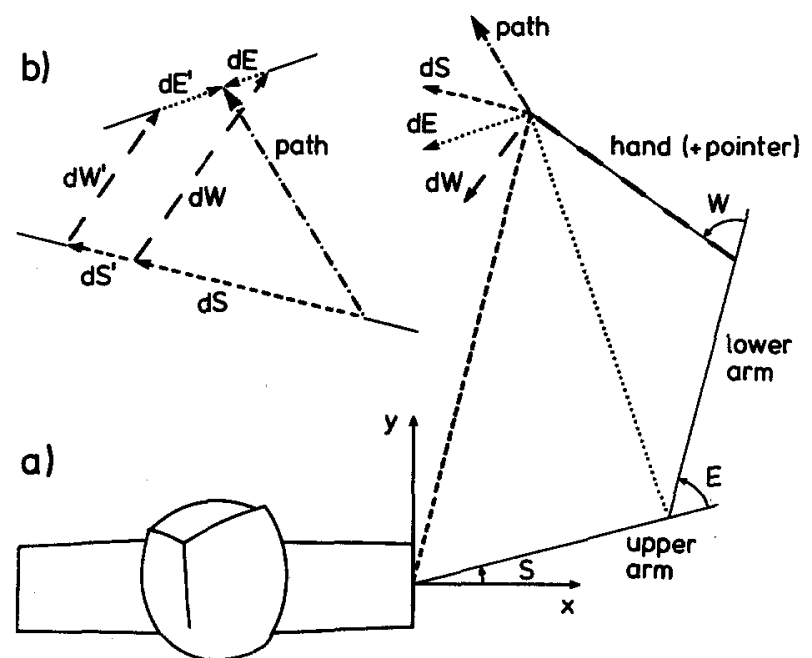

Fig. 1. a A schematic drawing of the arm in the horizontal plane. $S$ : shoulder angle, $E$ : elbow angle, $W$ : wrist angle. Workspace coordinates: $x$ lateral, $y$ frontal. The arm is shown by thin solid lines, the lever arms connecting the joints with the tip of the pointer are shown by interrupted lines. Rotations of these lever arms about incremental angle values move the tip of the pointer along the vectors $\mathrm{d} S, \mathrm{~d} E$, and $\mathrm{d} W$ shown in corresponding interrupted lines. b Two different sets of such vectors are shown which move the tip of the pointer to the same point in the workspace. According to the solution using the pseudoinverse those vectors are selected whose squares of the length give the smallest sum

ments between start and end point typically took up to $2 \mathrm{~s}$. The results shown here are from one subject. Qualitatively the same results were found in two other subjects.

\section{Results}

\subsection{Movements Along a Straight Line}

Preliminary experiments showed that not all movements were performed with straight paths (see Sect. 3.2 for details). To allow a simple comparison between the results of the earlier investigation of the static behavior (angle values at the end of the movement), at first the subjects were told to move the tip of the pointer along a straight line in the workspace which was marked by crosses on the table. Movements were performed parallel to the abscissa and parallel to the ordinate. Figure 2a shows the former in the workspace coordinates. Figure $2 b$ and $c$ shows the results in joint space coordinates. The abscissa in both diagrams is the elbow angle. The ordinate is the wrist angle in Fig. $2 b$ and the shoulder angle in Fig. 2c. The experiments were done in two ways either by moving the arm continuously from start to end points or by short intermediate stops at the marked points along the line. Only the data of the former experiment are presented 
a)
巳
0
음
0
$\frac{1}{2}$
$\vdots$
3
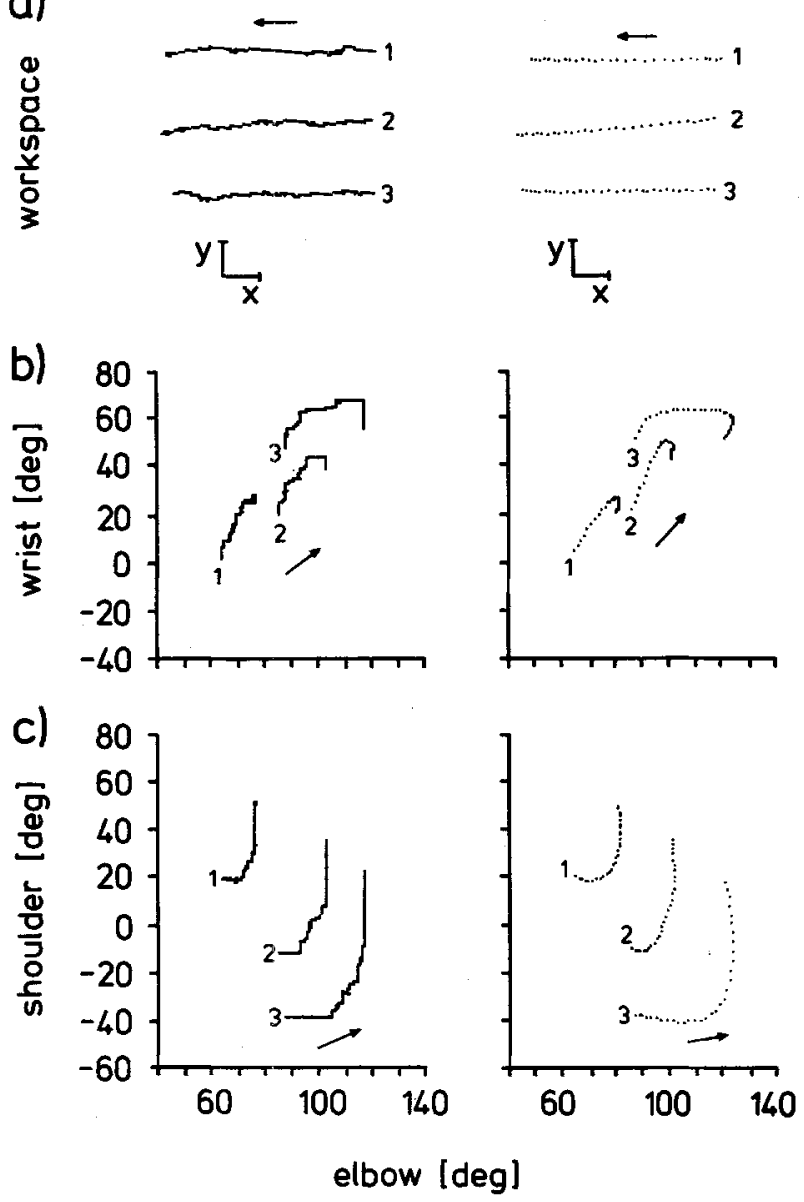

Fig. 2a-c. Movement of the arm when the start and end point are given and the path is prescribed as a straight line between start and end points. a the path of the tip of the pointer in workspace coordinates $x$ and $y$. The shoulder lies at the origin. $\mathbf{b}$ and $\mathbf{c}$, joint space representation. The abscissa is the elbow angle in both drawings. The ordinate is the wrist angle in $\mathbf{b}$ and the shoulder angle in $\mathbf{c}$. The arrows indicate the direction of movement. For the definition of the angles see Fig. 1. The right hand figures show the corresponding results of the model calculation

in the figure because no essential differences were found between the two experiments. This result shows that for a given point in the workspace the same combinations of angle values are used in both the dynamic and the static situation. As in the earlier investigation of static experiments the data were used to calculate those cost functions by which the results could be described with the least squares deviation (for details see E. Wischmeyer, in preparation).

\subsection{Movements Between Two Points with Free Choice of Path and of Joint Angles}

The following experiments were done to find out which joint angles and which workspace paths were chosen
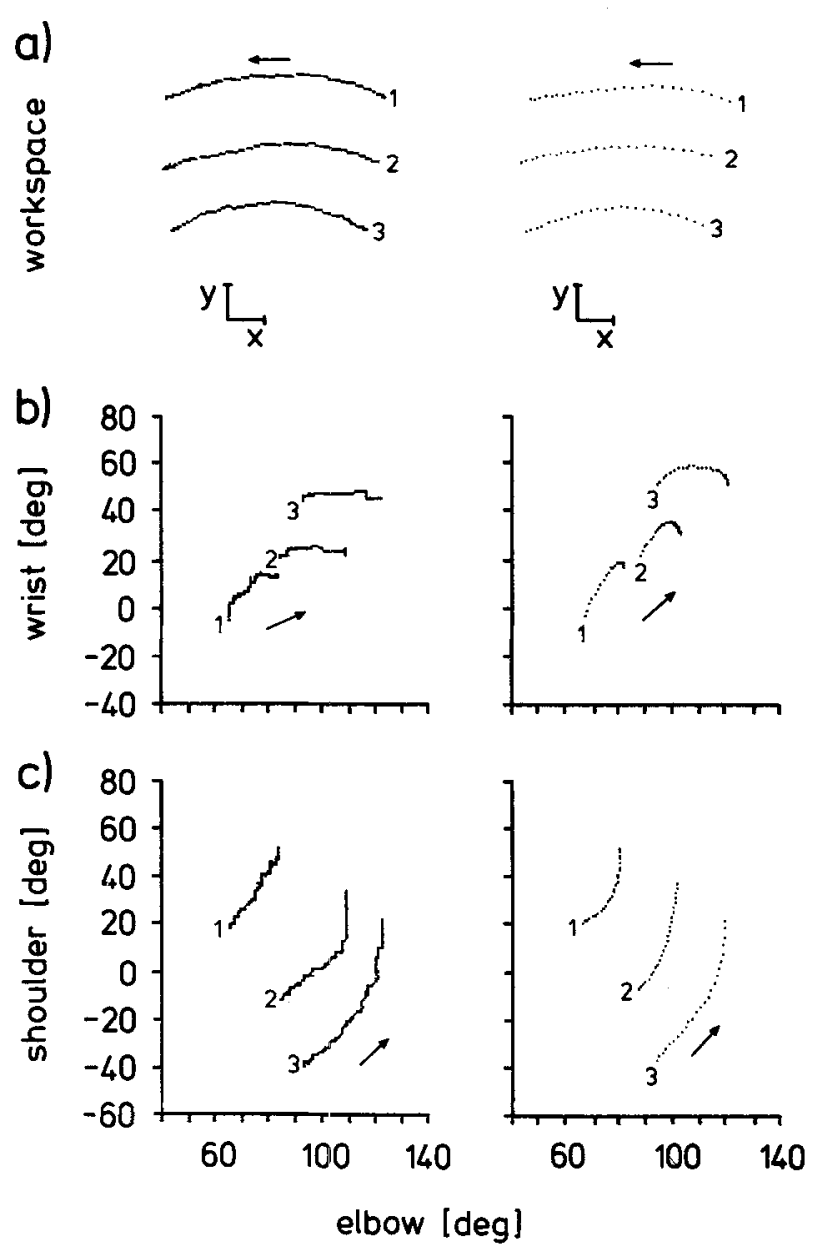

Fig. 3a-c. Movement of the arm as in Fig. 2 but when only start and end points are given and joint angles and path can be chosen deliberately. For further explanation see Fig. 2

when performing a comfortable movement between a given start point and a given end point. The results are shown in Figs. 3 and 4. The lines connecting start and end points of a movement are parallel to the $x$-axis of the workspace in Fig. 3 which can therefore directly be compared with those of Fig. 2. Figure 4 shows the corresponding results for movements parallel to the $y$-axis of the workspace. The lines connecting the start and end points in the experiments of Fig. 5 show an angle of $\mathbf{4 5}$ degrees relative to the workspace coordinates. These results show that in the workspace coordinates most paths are approximately straight lines but the trajectories in the joint space are generally curved. There are, however, some cases in the workspace where the paths are curved, particularly those shown in Fig. 3.

The time functions of the joint angle values are not shown but the qualitatively important features can be deduced from the joint space presentations. Most often the angle value follows a monotonic function i.e. it 

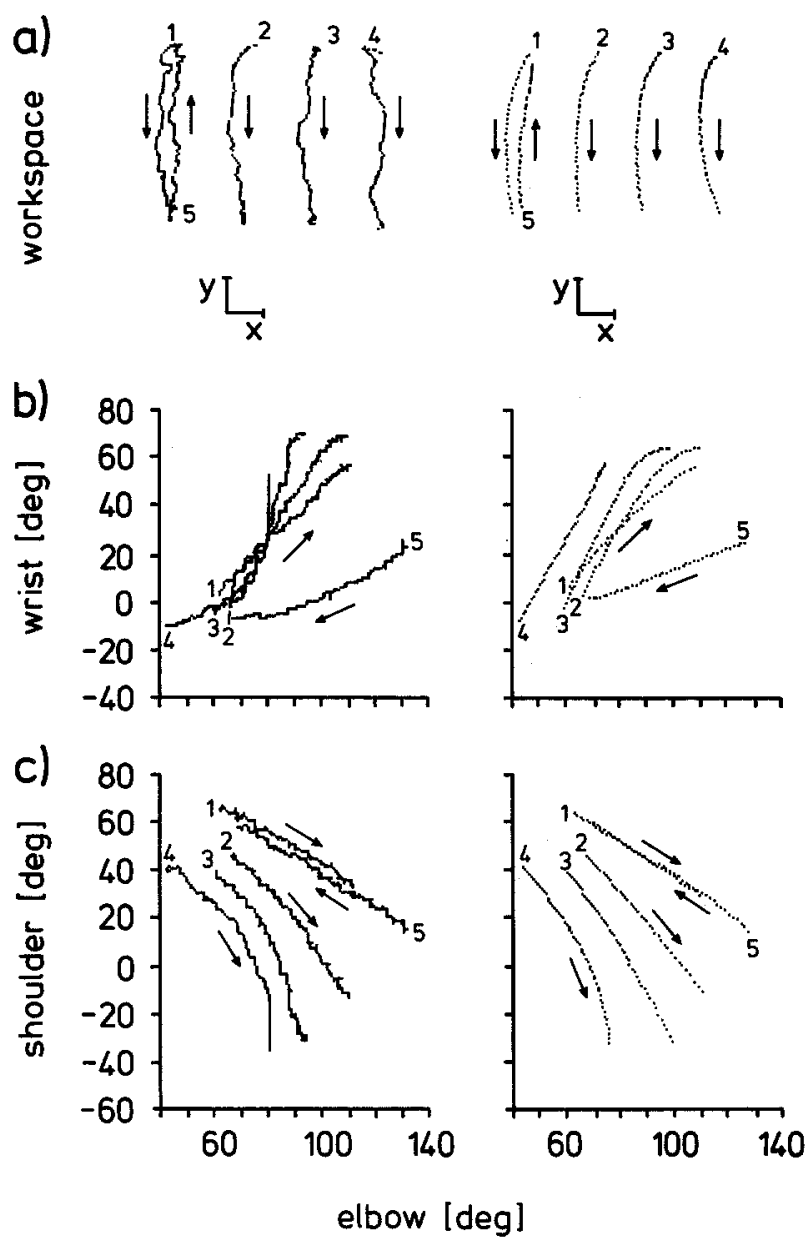

Fig. 4a-c. Movement of the arm with free choice of the path. The lines connecting start and end points are parallel to the $y$-axis of the workspace. For further explanation see Fig. 2

steadily increases or steadily decreases during the movement. This suggests that a simple rule underlies the control of the joint movement: acceleration by the agonist and then deceleration by the antagonist. However there also exist cases of non-monotonic movements (see Fig. 5, trace 2 for the elbow joint). In these cases the movement has to be controlled by a basic pattern of agonist, antagonist and finally again agonist activation. When the choice of the path is free, for the wrist joint movement is always monotonic, but in some cases for the shoulder angle and even more obviously for the elbow angle the direction of movement changes sign. It should be mentioned here that such a change of the direction of movement is often necessary to move the end effector along a straight line in the workspace. Comparison of the traces in Fig. 2 and Fig. 3 shows the difference in the joint space presentation of movements between the same points but with straight and curved paths. When the tip of the
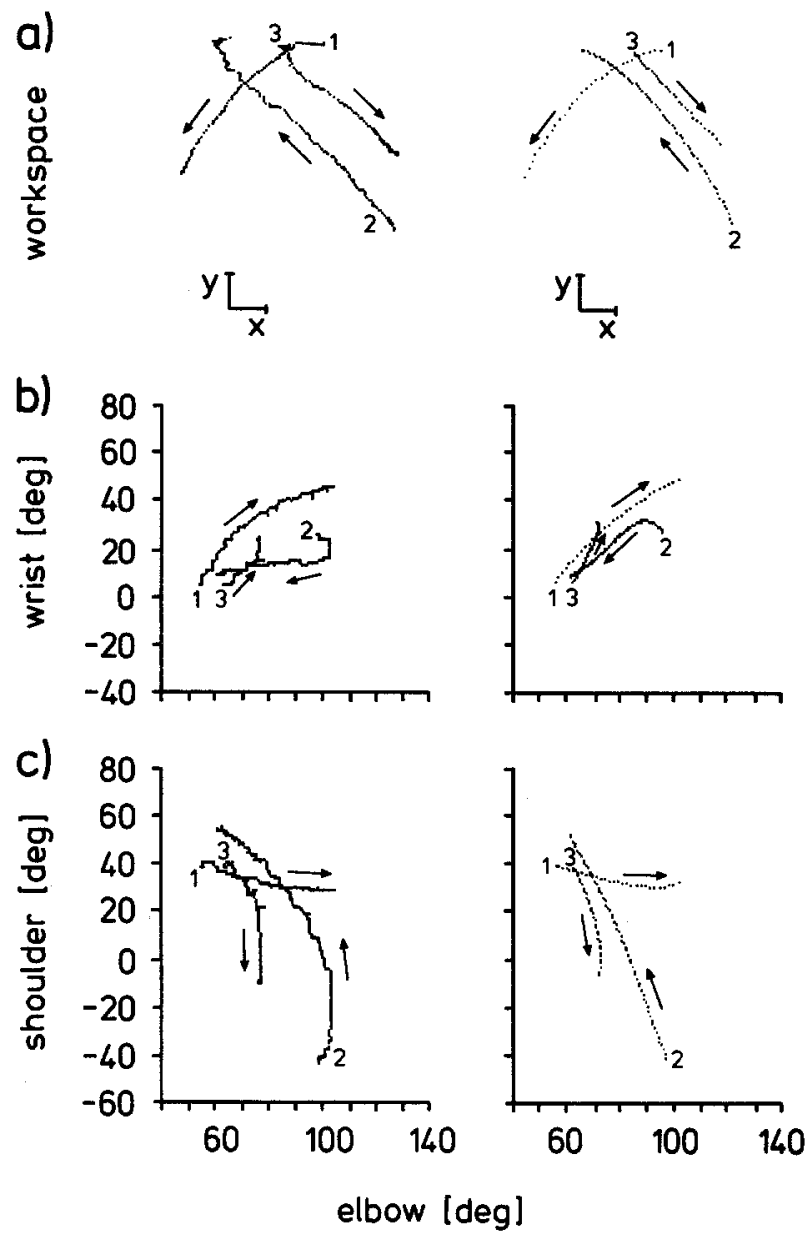

Fig. 5a-c. Movement of the arm with free choice of the path. The lines connecting start and end points show an angle of 45 degrees relative to the workspace coordinates. For further explanations see Fig. 2

end effector is constrained to move along a straight line (Fig. 2) then the wrist angle shows a non-monotonic movement. When the choice of the path is free (Fig. 3), a curved path is used which allows the wrist joint to perform a monotonic movement. Furthermore the curves in Fig. 3c (joint space) are less curved than those in Fig. 2c.

\subsection{Movements Between Two Points}

with Free Choice of Path

But Prescribed Joint Angles at the Start

In these experiments the subject was told to perform a comfortable movement between a given start point and a given end point. In contrast to the earlier experiments the joint angles at the start were chosen in an extreme and rather uncomfortable position. The results of some examples are shown in Figs. 6 and 7. 

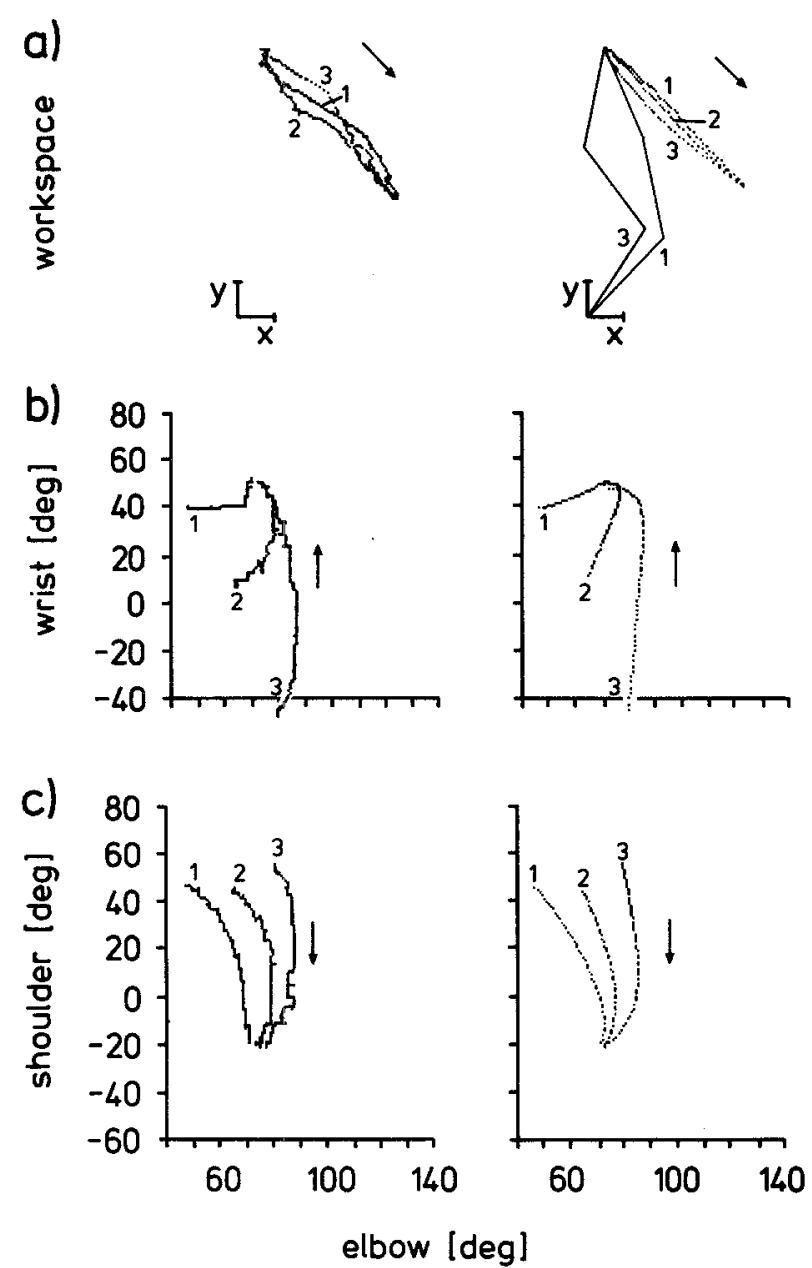

Fig. 6. Movement of the arm between two points with free choice of the path, but prescribed joint angles at the start. Trace 2 shows a movement with a "comfortable" starting position, traces 1 and 3 show movements where the arm at the beginning was held in an extreme position as shown by the inset. For further explanation see Fig. 2

The workspace representation includes an inset sketch of the positions of the arm at the beginning. In both figures trace 2 corresponds to the movement with the comfortable starting position. Figure 6 shows a case in which the normal movement showed a straight path. This is again, at least approximately, the case when the two uncomfortable starting positions were used. Figure 7 shows that the curvature of a curved path could be even stronger depending on the joint angles at the start.

These results show that for the same positions of the end effector in the workspace very different joint angle values may be adopted. Thus the angle values are not determined according to a fixed rule defined on the work space coordinates. They rather depend on the history, i.e. on the values the joint angles had at some previous time.
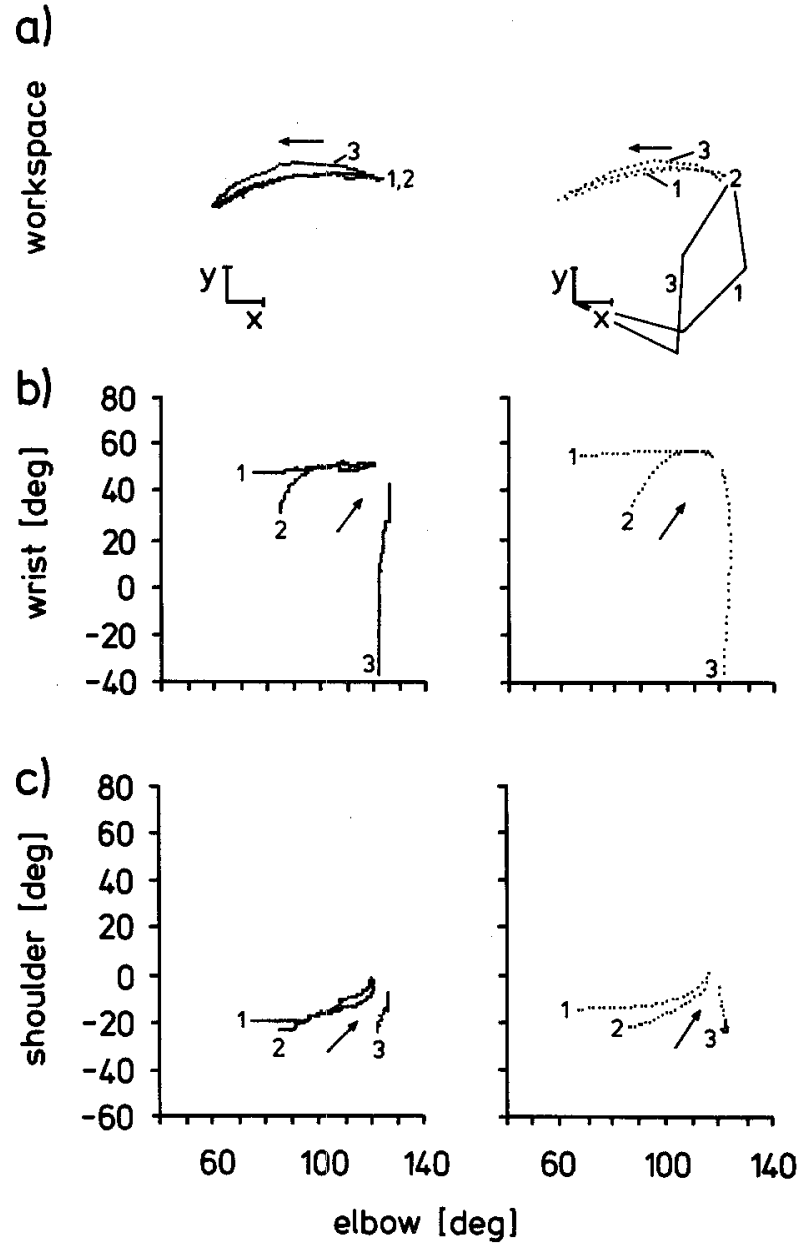

Fig. 7. Movements as shown in Fig. 6 but with other start and end points. For further explanation see Fig. 2

\section{Discussion}

Two questions are examined in this investigation: a) What do the paths look like and $b$ ) what might be the underlying mechanisms that produce the path? The results showed that the path for a comfortable movement between two points usually approximates a straight line of the end effector in the workspace. This agrees with findings of Morasso (1981), Soechting and Lacquaniti (1981), Abend et al. (1982), and Hollerbach and Flash (1982). Atkeson and Hollerbach (1985) only found curved paths for arm movements in a vertical plane. Therefore they speculated that the existence of curved paths might be caused by the action of gravity. Our results show that this is not the only explanation as we have found curved paths in some cases of horizontal movements.

What determines the path? One might speculate on the basis of minimizing the total cost value (Cruse 1986), that the path is chosen so that at each moment it attempts to keep the total cost value as small as 
possible. The minimum of the cost function for each joint presumably occurs at angles near the middle of the range. Thus according to this hypothesis the curves in the joint space coordinates should always tend to pass the points given by these coordinate values (e.g. about 0 degrees for the shoulder, 80 degrees for the elbow and 10 degrees for the wrist). The results show that this is not the case. Another hypothesis might be that the arm does not reach the minimum value but at least attempts to avoid positions with high total costs. This means that the arm moves along a curve with constant costs at least as long as it is not constrained for geometrical reasons to move to positions with higher costs. Alternatively, if the total cost of the end point position is smaller than that of the starting position, the arm should first move to a position with the cost value of the final position and then move along a curve with constant costs. However, the results did also not support this hypothesis either.

A third hypothesis which is ruled out as a sole explanation by these results is the so-called massspring hypothesis (Fel'dman 1974). This assumes that the values of the joint angles of the final position are calculated before the movement has started. The movement could then be performed so that each joint moves to its final angle independently of the other joints. Although this is an appealing hypothesis with several theoretical advantages (see Hollerbach and Atkeson 1986) it is not supported by the results. According to this hypothesis one should find nearly straight lines in the joint space presentation. This is generally not the case. As mentioned earlier, sometimes the movement of a joint even changes direction. This shows that the movement of the individual joints is not independent but that there is a superimposed control center which controls the timing of the individual muscles during the movement.

For movements in the vertical plane Hollerbach et al. (1986) found curved paths. From these results they concluded the following underlying principle. Normally, a movement in joint space is performed so that a straight line results in the workspace. But nonmonotonic joint movement is avoided by delaying the movement of one of the joints by a given amount of time [see also Kaminski and Gentile (1986) for a similar hypothesis which is however not in agreement with our findings]. The value of this additional control parameter depends on the location of start and end points. In the joint space this would lead to polygons of vertical and horizontal sections and oblique straight lines. However, we actually found non-monotonic joint movements in the elbow (Fig. 5) and shoulder joints (not shown). Thus delaying the movement of selected joints is not sufficient to describe these movements. The situation is further complicated by the fact that not only the position of the start point but also the angle values at the starting position determine the motor control pattern during the movement (Figs. 6 and 7). Therefore the positions of the start and end points alone do not provide enough information to determine the control parameters.

The aim of the control system of the human arm seems to be to produce straight paths of the end effector in the workspace. Particularly the nonmonotonic joint movements (Fig. 6, trace 2 and 3 ) very strongly support this suggestion. However, as mentioned above, there are also cases of curved paths. These cannot be traced back to inertia effects because $a$ ) they also appear when using very slow movements and $b$ ) they appear only in some of the joint angle ranges and not in others. Another possible cause for the appearance of the curved paths can also be excluded. One might think of the paths always being planned and performed as straight lines in some topologically distorted space rather than that described by cartesian coordinates. Two arguments oppose this assumption. First, if the curved paths of Fig. 3 are subject to a continuous distortion of the space, this distortion should also effect the paths described in Fig. 4, Fig. 5 and in trace 2 of Fig. 6. However, with the exception of trace 1 in Fig. 5 they seem to follow quite straight lines. Second, the results of Figs. 6 and 7 show that differently curved paths result for the same starting and end points depending on the values of the joint angles at the starting position. Thus, the path does not follow a straight line in a space subject to a constant distortion.

The results show that the path depends on the joint angles at the start and on the position of the end point in the workspace. One might argue that, instead of knowledge about the position of the end point, knowledge about the direction of the end point might be sufficient. The curved paths, however, show that both direction and distance between the two points determine the form of the path. The paths from a given start point to end points which lie in the same direction but at different distances differ from the beginning (Fig. 8). This means that the form of the path is not only a consequence of local control laws but also of global control laws. A proposal showing how the path might be calculated is given in the last section.

How are the individual joints controlled? The results of Fig. 2 suggest that after the determination of the position of the end effector the joint angles are determined by means of the minimum cost principle. The results presented in Fig. 6 show however that the angle values can be different even for approximately the same path of the end effector. If the minimum cost principle alone was responsible, then after the start from an "uncomfortable" arm position the angle 


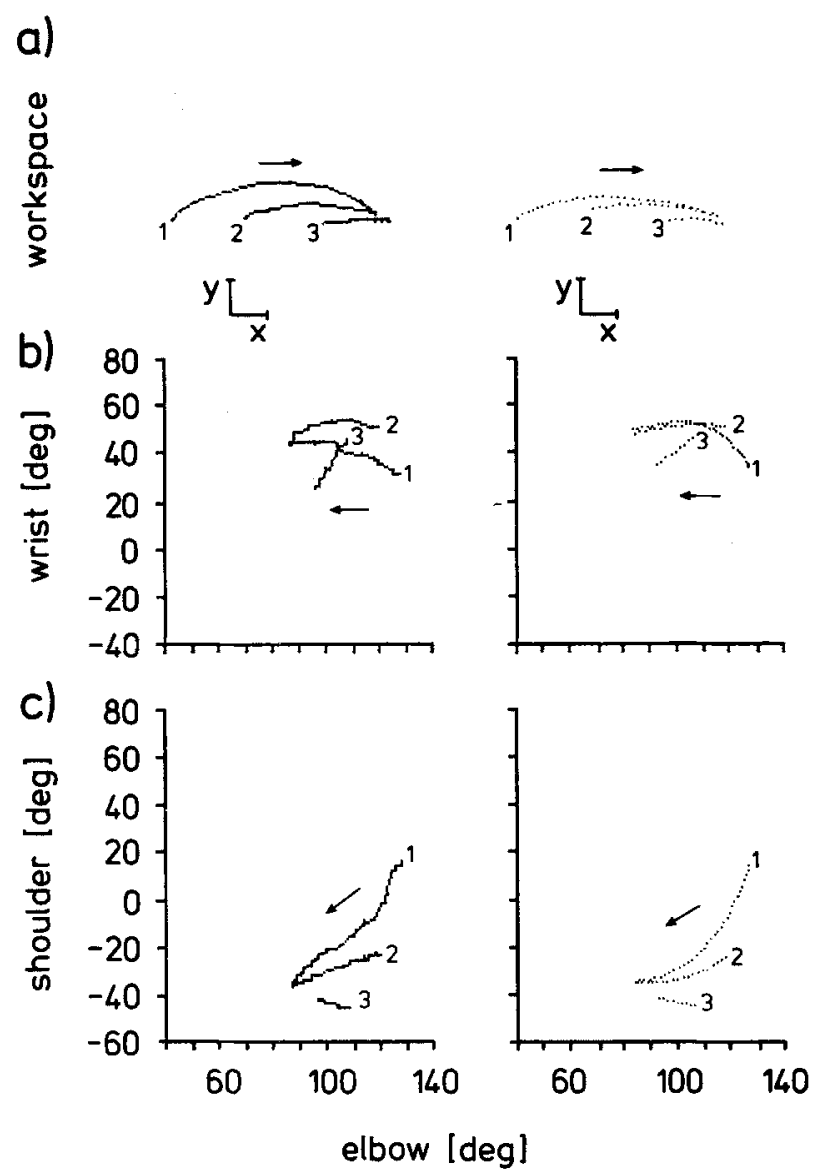

Fig. 8. Movements of the arm from three different starting points to the same end point. The choice of the path is left open to the subject. For further explanation see Fig. 2

values should immediately switch to a "comfortable" position without moving the tip of the end effector and then continue to move the end effector according to the minimum cost principle. The actual arm, however, moves the tip of the end effector immediately after the start and seems to approach angle values which correspond to the minimum cost principle only gradually during the ongoing movement. Thus, the movement of the joints in the direction of a comfortable angle arrangement and the movement along the prescribed path seem to be performed in parallel. If there is enough time during the movement, the angle values near the end of the movement correspond quite well to those of the minimum cost principle. In other cases which are not shown here, even the joint angle values at the end of the movement were different depending on the starting arm position and so revealed the history dependence of the control system.

\section{Model}

These considerations lead to the following model which is simulated in Basic and provides the qualitative properties found in the experiments with the human arm. As the experimental results show, the path of the end effector usually is a straight line in the workspace between start point and end point. Sometimes, however, curved paths appear.

For the first stage a model will be described which is only able to produce straight paths. How are the joint angles chosen? For a non-redundant system the joint angles are completely determined by geometrical constraints once the form of the path is fixed. In the case of a redundant system a mechanism is necessary to reduce the number of degrees of freedom. To make the discussion easier, in Fig. 1 a the three lever arms which connect each joint with the end effector, i.e. the tip of the pointer, and the three vectors which describe the movement of the end effector as the result of incremental angle changes in the three joints are shown. Corresponding lever arms and vectors are drawn with the same symbols. As discussed earlier two conditions seem to determine the angle values. First, the changes of the angles should follow the minimum cost principle (within the geometrical possibilities). Second, these changes should allow the end effector to move along the path. Both conditions cannot be satisfied at the same time. Therefore a compromise is required that is implemented in the model as follows. To fulfill the second condition, the incremental angle changes $d S$, $d E$, and $d W$ (see Fig. 1) are calculated so that the sum of the squared vector lengths is a minimum. This means that all three vectors have, as far as geometrically possible, about the same length. This is quite similar to the often discussed application of the pseudoinverse (see e.g. Hollerbach and Suh 1985, for excellent, concise summary). To establish the first condition the vector lengths are weighted by the value of the corresponding cost functions. In the earlier paper (Cruse 1986), the cost functions were $U$-shaped to obtain only positive values. In the present model, one of the two branches was given a negative sign, thus introducing the direction of the deviation from the intercept into the form of the cost function. This intercept corresponds to the minimum of the cost function used in the earlier paper. This model provided a qualitative description of those experiments showing straight paths. At this stage the model very much resembles proposals made by Yoshikawa (1984).

What might cause the deviation from the straight path in the other experiments? One might assume that curved paths appear when the performance of a straight line would require very high cost values in one or more joints and they could be avoided by adopting the curved path. Although this might be true in such trivial cases, when the joint arrived at its mechanical limits, this could not be an explanation for our results as this situation does not occur in our experiments. The curved paths found here, appear for angle values in 
both high cost and low cost ranges. Thus, the existence of curved paths cannot be explained by the hypothesis to avoid very high cost values. The following observation leads to an alternative hypothesis. Curved paths seem to appear for those movements in which the trajectories in the joint space would have required to be strongly nonlinear. Such a nonlinearity requires a more complicated timing of the individual muscles. This suggests that the strategy producing curved paths might be adopted in order to simplify the task of the control system. The most easy way to control the movement of the arm would be to plan the path in joint coordinates so that a straight line in the elbowshoulder graph and the wrist-elbow graph would occur (which corresponds to the mass-spring principle discussed earlier). The data do not show such a result. The control system rather seems to use a compromise between a straight line in the joint space and a straight line in the workspace.

Such a compromise is implemented in our model by extending the first stage model in the following way: the incremental angle changes calculated as described above are added to changes which correspond to the mass-spring hypothesis. The contribution of the latter is high at the beginning of the movement but declines to zero at the end. Therefore the prior estimation of the joint angles for the final arm position, which is a necessary assumption for the mass-spring hypothesis, is not critical. These values need only be approximate. The results of the model calculation are shown in Figs. 2-8 together with the experimental results to allow direct comparison. A more detailed description of the model will be given elsewhere. In general, this hypothesis agrees with the proposal of Hollerbach et al. (1986) who for movements in the vertical plane also state an intermediate strategy between straight lines in the joint space and straight lines in the workspace. These two hypotheses differ in the following respect. In our hypothesis, calculation of the actual path and of the incremental angle changes is done on the local level. According to the hypothesis of Hollerbach et al. both, determination of the form of the path and the calculation of the details of the temporal order of muscle activation are done on the global level. In their case the problem of redundancy is not addressed in detail.

An alternative model was also tested and gave better results: before starting the movement, a decision was made whether to follow a straight path (corresponding to the first stage model) or the curved path strategy (extended model). A working algorithm for this decision was found but seems to be too artificial and is therefore not discussed here in detail.

In summary, the control system of the movement of the redundant human arm can be interpreted as compromise between four requirements, a) an equal contribution of all joints to the movement, $b$ ) minimization of the static costs by means of the cost functions, c) minimizing the inertial forces acting at the tip of the end effector by following a straight workspace path, and $d$ ) simplifying the pattern of muscle activity (in particular by avoiding non-monotonic joint movements as far as possible).

Acknowledgements. We would like to express our thanks to Dr. J. Dean for helpful discussions and Dr. D. Graham for proof reading the English manuscript.

\section{References}

Abend W, Bizzi E, Morasso P (1982) Human arm trajectory formation. Brain 105:331-348

Atkeson CG, Hollerbach JM (1985) Kinematic features of unrestrained vertical arm movements. $J$ Neurosci $5: 2318-2330$

Cruse H (1986) Constraints for joint angle control of the human arm. Biol Cybern 54:125-132

Fel'dman AG (1974) Change of muscle length as a consequence of a shift in an equilibrium of muscle load system. Biophysics 19:544-548

Hollerbach JM, Atkeson CG (1986) Characterization of jointinterpolated arm movements. In: Heuer $\mathrm{H}$, Fromm $\mathbf{G}$ (eds) Generation and modulation of action patterns. Springer, Berlin Heidelberg New York, pp 41-54

Hollerbach JM, Flash T (1982) Dynamic interactions between limb segments during planar arm movement. Biol Cybern 44:67-77

Hollerbach JM, Moore SP, Atkeson CG(1986) Workspace effect in arm movement kinematics derived by joint interpolation. In: Gantchev G, Dimitrov B, Gatev P (eds) Motor control. Plenum Press, New York

Hollerbach JM, Suh KC (1985) Redundancy resolution of manipulators through torque optimization. In: Proceedings of the 1985 IEEE International Conference on Robotics and Automation, pp 1016-1021. Computer Soc. Press, Silver Spring

Kaminski T, Gentile AM (1986) Joint control strategies and hand trajectories in multijoint pointing movements. $\mathrm{J}$ Mot Behav 18:261-278

Morasso P (1981) Spatial control of arm movements. Exp Brain Res 42:223-227

Soechting JF, Lacquaniti F (1981) Invariant characteristics of a pointing movement in man. $J$ Neurosci 1:710-720

Yoshikawa T (1984) Analysis and control of robot manipulators with redundancy. In: Brady M, Paul R (eds) Robotics research. The First International Symposium. MIT Press, Cambridge, Mass, London, pp 735-747

Received: April 4, 1987

Prof. Dr. H. Cruse

Fakultät für Biologie

Universität Bielefeld

Postfach 8640

D-4800 Bielefeld 1

Federal Republic of Germany 